\title{
Determination of velocity and stress discontinuities in quasi-static granular flows
}

\author{
Yong-Hong $\mathrm{Wu}^{*} \quad$ Roger Collinson*
}

(Received 7 August 2000)

\begin{abstract}
In quasi-static flows of granular materials, there often exist stress and velocity discontinuities in the material. However, existing numerical techniques are generally not capable of modelling these discontinuities satisfactorily. In this paper, we develop a sophisticated numerical technique for simulating the quasi-static flow of granular materials in the presence of stress discontinuities. Based on the double-shearing
\end{abstract}

* School of Mathematics and Statistics, Curtin University of Technology, Perth, WA 6845, Australia. mailto:yhwu@cs.curtin.edu.au

${ }^{0}$ See http://anziamj.austms.org.au/V42/CTAC99/Wu1 for this article and ancillary services, (C) Austral. Mathematical Soc. 2000. Published 27 Nov 2000. 
model, the governing equations consist of four highly nonlinear partial differential equations with two families of characteristic lines. We firstly transform the partial differential equations into a set of ordinary differential equations using the method of characteristics. Then, a computation scheme is developed to determine the characteristic lines, the stress discontinuity lines and the stress and velocity fields. The method is then applied to study the flow of granular materials in silos.

\section{Contents}

1 Introduction

C1560

2 Basic field equations

C1564

3 Determination of stress discontinuity lines

C1566

4 Numerical example

C1571

5 Conclusions

C1571

References

C1576 


\section{Introduction}

A granular material can be defined as a collection of a large number of discrete solid particles in contact with each other. Some examples of granular materials are mineral products, ore, sand, soil, grains and chemical powders. The study of granular flows is important to the solution of a wide range of scientific and technological problems related to material handling, pneumatic transport, flows of slurry in pipes, mineral processing, stability of open pits, sediment transport in rivers, and etc.

Granular materials are neither solid nor liquid as commonly understood. Under certain conditions, a granular material can behave like a solid. However, when the stress state satisfies the yield condition, the so-called quasistatic flow occurs and the material yields along stress characteristics and flows as blocks, each consisting of many granules, moving relative to one another along thin slip lines. If the deformation occurs rapidly enough, the so-called rapid flow regime occurs and the entire mass of material moves independently such that grains are in relative motion with even their nearest neighbours. Due to this dual nature, granular flows are extremely complex and the existing theories of solid mechanics and fluid dynamics are not directly applicable to this subject.

Over the last few decades, extensive research has been carried out to study the flow of granular materials. Early research mainly focused on experimental investigation and deriving approximate analytical methods and 
empirical formulae suitable for engineering design $[6,11,12,14]$. In the last two decades, many researchers have investigated granular flows theoretically and a number of mathematical models have been developed for analysis. Essentially, these models can be classified into two categories: discrete models and continuum models.

Discrete models determine the overall macroscopic mechanics behaviour of granular flows by following the motion of individual particles either precisely or statistically. The major type of discrete models is the so-called discrete element model, which is based on the molecular dynamics and was first applied to simulate granular flows by Cundall [7] and later by others [5, 20]. Two different types of discrete elements have been developed, namely the hard particle model [4] and the soft particle model [19]. The hard particle model generally is only applicable to rapid flows with low bulk density. While the soft particle model is applicable to both rapid and slow flows. Statistical mechanics methods [1] have also been incorporated into discrete element models and in this new kind of models, the flow of particles is simulated by either the Markov process [15] or the Monte Carlo method [10].

The bulk of the literature on granular flow is concerned with the continuum models. In such kind of models, the discrete nature of the material and details of inter-particle interactions are not considered. The constitutive nature is hidden in phenomenological coefficients involved in the constitutive equations. The macroscopic behaviour of granular flows is then described by differential forms of mass, momentum and energy conservation laws. Over the last couple of decades, two types of theories have been developed, namely 
the statistical collision theory for rapid flows and the frictional plasticity theory for slow flows. The collision theory is based on micro-mechanics analysis of particle collision and statistical averaging [2, 3, 8]. The frictional plasticity theory is based on the assumption that the material flows according to a constitutive law if the stress state satisfies a yield condition. It is generally believed that the plasticity theory can be used to model slow granular flows. The equations, which distinguish granular flows from other kinds of plasticity flows, are the yield condition and the constitutive equations. Many different yield conditions have been established $[9,13]$ and two different theories have been proposed to derive the constitutive equations for granular materials, i.e, the conventional plastic flow rule theory $[16,17]$ and the so-called double-shearing theory originated by Spencer [18] and developed by Spencer and others. In this paper, we study the quasi-static granular flow using the double shearing theory. The basic form of the double-shearing flow theory is based on the Mohr-Coulomb yield condition. If the stress state in a granular material reaches the yield condition, flow occurs by shearing along two families of planes, namely the $\alpha$ - and $\beta$ - characteristics. These two planes are inclined at angles $\pm \varepsilon(\varepsilon=\pi / 4+\phi / 2)$ to the direction of the principal stress $\sigma_{1}$ where $\phi$ is the angle of material internal friction, as shown in Figure 1. The double shearing theory assumes that the complete deformation at any point of the material consists of two shearing motions occurring simultaneously on the $\alpha$ - and $\beta$-lines and which are superposed one on another. More specifically, in the rectangular Cartesian coordinate systems as shown 


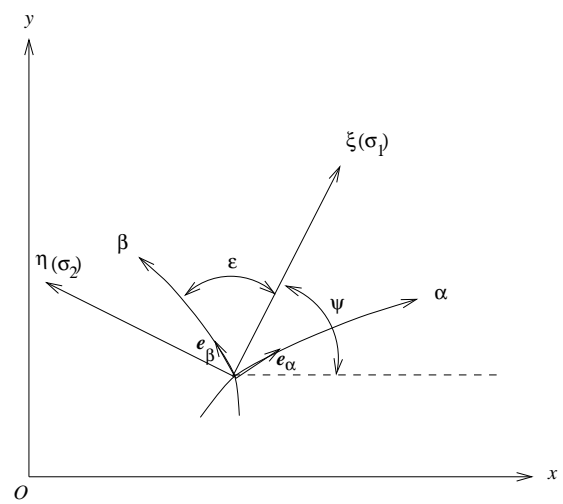

Figure 1: Coordinate system, $\alpha$ - and $\beta$ - lines.

in Figure 1, the double-shearing assumption can be formulated by

$$
\frac{\partial \mathbf{v}}{\partial s_{\alpha}}=a \mathbf{e}_{\beta}, \quad \frac{\partial \mathbf{v}}{\partial s_{\beta}}=b \mathbf{e}_{\alpha},
$$

where the first set of coordinate system $O_{x y}$ is fixed in space while the second set $O \xi \eta$ is fixed on the particle and is in motion relative to the first set with rotating speed $\Omega$ and translational velocity $\left(v_{x}, v_{y}\right), s_{\alpha}$ and $s_{\beta}$ are measured along the slip-line, $\mathbf{e}_{\alpha}$ and $\mathbf{e}_{\beta}$ denote unit vectors along the direction of $\alpha$ and $\beta$ respectively. The rest of the paper is organised as follows. In the following section, the equations governing the stress field and velocity field are presented. In Section 3, a numerical method for the determination of the stress discontinuity lines is developed. In Section 4, the method is used to 
study the flow of granular materials through silos. Conclusions are made in Section 5.

\section{Basic field equations}

Consider the plane flow in the Oxyz rectangular Cartesian coordinate system with the $x$-axis being vertical upward. The equations of motion for the quasistatic flow are

$$
\frac{\partial \sigma_{x x}}{\partial x}+\frac{\partial \sigma_{x y}}{\partial y}-\rho g=0, \quad \frac{\partial \sigma_{x y}}{\partial x}+\frac{\partial \sigma_{y y}}{\partial y}=0 .
$$

If $\sigma_{1}$ and $\sigma_{2}$ denote the maximum and minimum principal stress components namely $\left(\sigma_{1} \geq \sigma_{z} \geq \sigma_{2}\right)$ and if we assume that the direction of $\sigma_{1}$ makes an angle of $\psi$ with the $x$-axis, then we have

$$
\sigma_{x x}=-p+q \cos 2 \psi, \quad \sigma_{y y}=-p-q \cos 2 \psi, \quad \sigma_{x y}=q \sin 2 \psi,
$$

where $p$ and $q$ are respectively

$$
p=-\frac{\sigma_{1}+\sigma_{2}}{2}, \quad q=\frac{\sigma_{1}-\sigma_{2}}{2} .
$$

Suppose that the material is cohesionless and obeys the Coulomb yield criterion. Then, the stress state satisfies

$$
q=p \sin \phi,
$$


where $\phi$ is a material parameter, namely the angle of internal friction. On substituting equations (3) into equations (2), we can deduce in the usual way the stress equations in terms of the arc lengths $s_{\alpha}$ and $s_{\beta}$ along the $\alpha$ and $\beta$ characteristics defined respectively by

$$
\begin{aligned}
& \frac{d y}{d x}=\tan (\psi-\varepsilon) \quad-\alpha \text { line } \\
& \frac{d y}{d x}=\tan (\psi+\varepsilon) \quad-\beta \text { line }
\end{aligned}
$$

Thus (2) becomes

$$
\begin{aligned}
\frac{\partial p}{\partial s_{\alpha}}+2 p \tan \phi \frac{\partial \psi}{\partial s_{\alpha}} & =-\frac{\rho g}{\cos \phi} \sin (\psi+\varepsilon), \\
\frac{\partial p}{\partial s_{\beta}}-2 p \tan \phi \frac{\partial \psi}{\partial s_{\beta}} & =\frac{\rho g}{\cos \phi} \sin (\psi-\varepsilon) .
\end{aligned}
$$

These two equations together with equations (6) and (7) constitute a system of four equations for the determination of the characteristic field and the $p$ and $\psi$ values along the characteristic lines.

In order to calculate the velocity field corresponding to the stress field, we adopt the so-called double shearing assumption as formulated in (1). The velocity equations based on this assumption have been shown to be

$$
\frac{\partial u}{\partial x}+\frac{\partial v}{\partial y}=0
$$

$\left(\frac{\partial u}{\partial x}-\frac{\partial v}{\partial y}\right) \sin 2 \psi-\left(\frac{\partial v}{\partial x}+\frac{\partial u}{\partial y}\right) \cos 2 \psi=\sin \phi\left(\frac{\partial u}{\partial y}-\frac{\partial v}{\partial x}+2 u \frac{\partial \psi}{\partial x}+2 v \frac{\partial \psi}{\partial y}\right)$. 


\section{Determination of stress discontinuity lines}

Consider the stress state around the discontinuity line as shown in Figure 2. Under steady state condition, the normal stress $\sigma_{n n}$ and shear stress $\sigma_{n t}$ must be continuous, namely

$$
\sigma_{n n}^{+}=\sigma_{n n}^{-}, \quad \sigma_{n t}^{+}=\sigma_{n t}^{-}
$$

where superscripts + and - refer to regions on either side of the discontinuity line as shown in Figure 2.

Suppose the normal $\mathbf{n}$ of the discontinuity line $L$ is inclined at an angle $\eta$ to the $x$-axis as shown in Figure 2. Then on either side of the discontinuity line,

$$
\begin{gathered}
\sigma_{n n}=\frac{\sigma_{x x}+\sigma_{y y}}{2}+\frac{\sigma_{x x}-\sigma_{y y}}{2} \cos 2 \eta+\sigma_{x y} \sin 2 \eta, \\
\sigma_{n t}=\sigma_{x y} \cos 2 \eta-\frac{\sigma_{x x}-\sigma_{y y}}{2} \sin 2 \eta,
\end{gathered}
$$

where for simplicity, we have dropped the superscripts \pm from the quantities $\sigma, p, q, \psi$ and $\eta$. Substituting (13)-(14) into (12) and using (3) and (5), we have

$$
\begin{gathered}
p^{+}\left[-1+\sin \phi \cos \left(2 \psi^{+}-2 \eta\right)\right]=p^{-}\left[-1+\sin \phi \cos \left(2 \psi^{-}-2 \eta\right)\right] \\
p^{+} \sin \left(2 \psi^{+}-2 \eta\right)=p^{-} \sin \left(2 \psi^{-}-2 \eta\right) .
\end{gathered}
$$




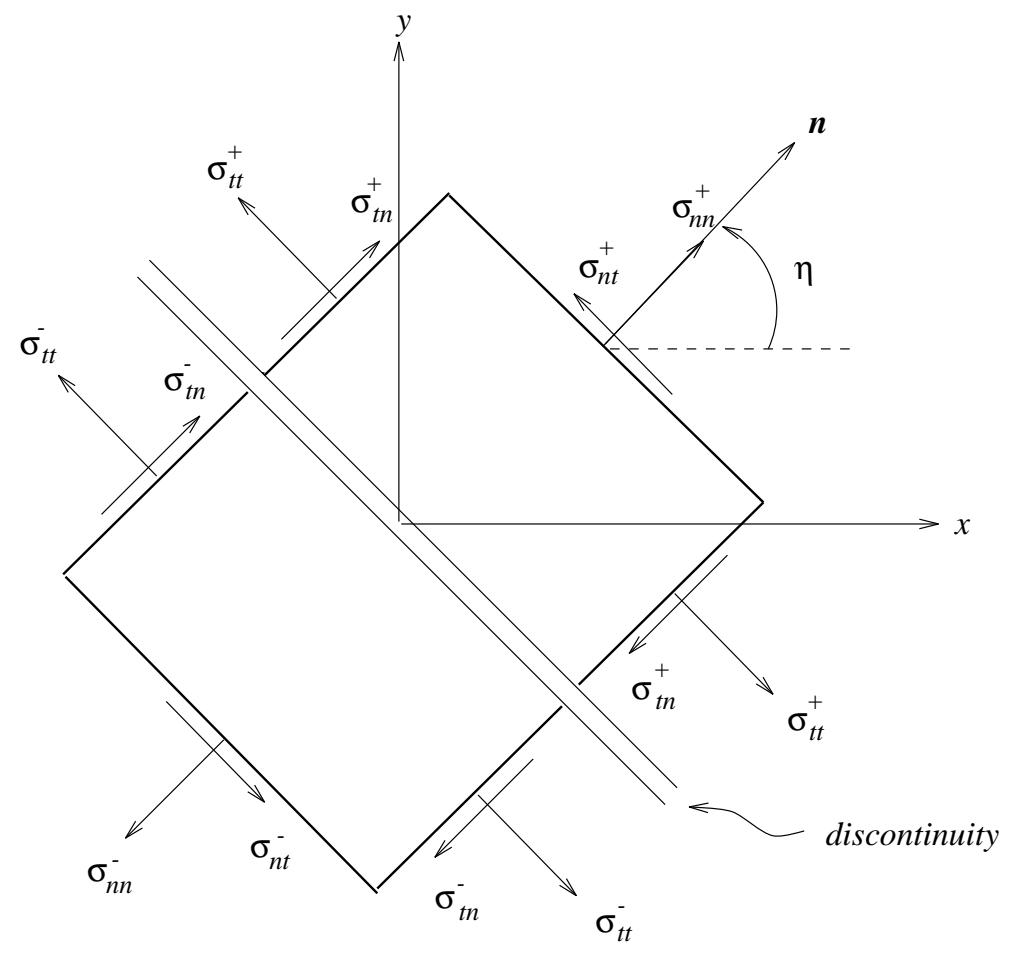

Figure 2: Forces acting on an element in the presence of discontinuity. 
These are the contact equations for the discontinuity line $L$ defined by

$$
\frac{d y}{d x}=\tan \zeta=\tan \left(\eta+\frac{\pi}{2}\right) .
$$

Depending on the boundary conditions, discontinuity lines can be classified into $\alpha$ - and $\beta$ - discontinuity lines. In the rest of this section, we demonstrate the determination of the $\alpha$ - discontinuity line.

Figure 3 shows the essential feature of an $\alpha$ - discontinuity line $L_{\alpha}$ defined by (17). Suppose that solutions of $p^{+}, \psi^{+}$on the '+' side have been obtained and that the $\alpha$-line from the '-' side intersects the discontinuity line at point $R$ with coordinates $(x, y)$, then there are five unknowns at point $R$, namely $x, y, p^{-}, \psi^{-}$and $\eta(x, y)$. The equations governing these unknowns are the discontinuity contact equations (15)-(16), the discontinuity line equation (17), the $\alpha$-characteristic line equation (6) and the stress equations along the $\alpha$-characteristics (8).

Let $D$ and $p$ denote a previously calculated point on the discontinuity line and the $\alpha$-line respectively. Then from the discontinuity line equation (17), the $\alpha$-characteristic line (6) and the stress equation along $\alpha$ (8), we have

$$
\begin{aligned}
& y-y_{D}=\tan \left(\eta+\frac{\pi}{2}\right)\left(x-x_{D}\right), \\
& y-y_{p}=\tan \left(\psi^{-}-\varepsilon\right)\left(x-x_{p}\right),
\end{aligned}
$$

$$
\cos \left(\psi^{-}-\varepsilon\right)\left(p^{-}-p_{p}\right)+2 \tan \phi \cos \left(\psi^{-}-\varepsilon\right) p^{-}\left(\psi^{-}-\psi_{p}\right)
$$




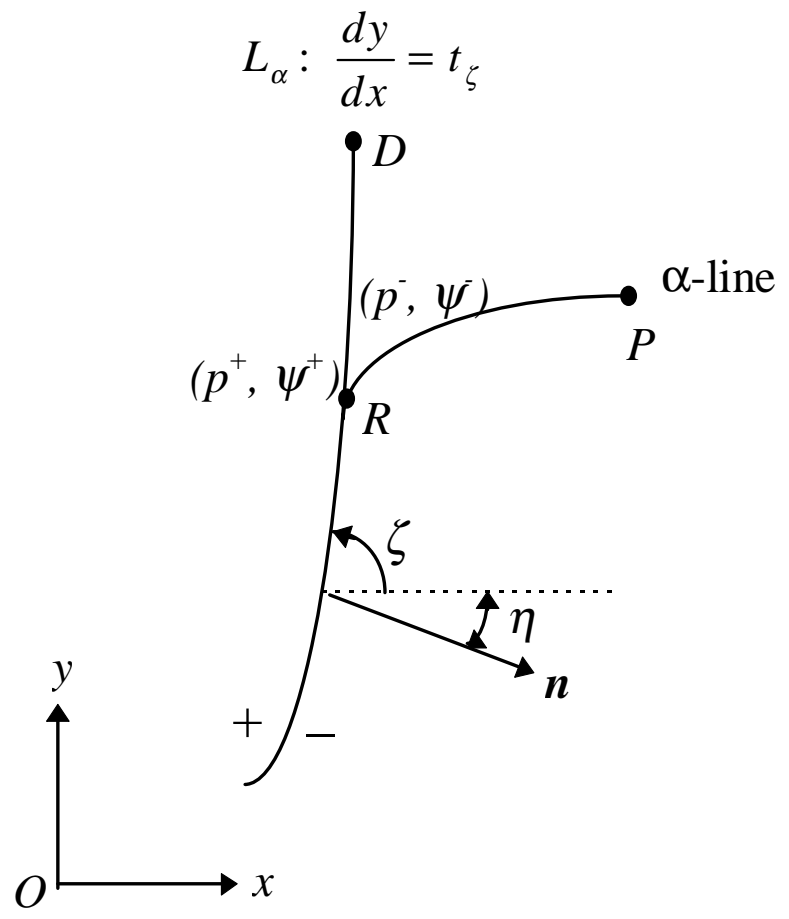

Figure 3: $\alpha$-discontinuity 


$$
=-\frac{\rho g}{\cos \phi} \sin \left(\psi^{-}+\varepsilon\right)\left(x-x_{p}\right) .
$$

Further, from equation (16), we have

$$
p^{-}=\frac{p^{+} \sin \left(2 \psi^{+}-2 \eta\right)}{\sin \left(2 \psi^{-}-2 \eta\right)} .
$$

Solving (18) and (19) yields

$$
\begin{gathered}
x=\frac{x_{p} \tan \left(\psi^{-}-\varepsilon\right)-x_{D} \tan \left(\eta+\frac{\pi}{2}\right)+y_{D}-y_{p}}{\tan \left(\psi^{-}-\varepsilon\right)-\tan \left(\eta+\frac{\pi}{2}\right)}, \\
y=\frac{\left[\left(x_{p}-x_{D}\right) \tan \left(\psi^{-}-\varepsilon\right)-y_{p}\right] \tan \left(\eta+\frac{\pi}{2}\right)+y_{D} \tan \left(\psi^{-}-\varepsilon\right)}{\tan \left(\psi^{-}-\varepsilon\right)-\tan \left(\eta+\frac{\pi}{2}\right)} .
\end{gathered}
$$

Substituting (21), (22) and (23) into (15) and (20) yields a system of two nonlinear equations in terms of two unknowns $\eta$ and $\psi^{-}$, namely

$$
\begin{aligned}
& F_{1}\left(\eta, \psi^{-}\right)=0, \\
& F_{2}\left(\eta, \psi^{-}\right)=0 .
\end{aligned}
$$

Newton's method can then be used to solve (24)-(25) for $\eta$ and $\psi^{-}$. Once $\eta$ and $\psi^{-}$are determined, $x, y$ and $p^{-}$can be calculated from (22), (23) and (20) respectively.

Similar formulae have also been developed for the determination of the $\beta$ - discontinuity lines. The corresponding velocity field is calculated within each computational domain separately using the method in [6]. 


\section{Numerical example}

The numerical scheme developed has been applied to study the stress distribution and velocity pattern of granular flows in hoppers in the presence of stress discontinuity. Figures $4-7$ show the characteristic mesh, stress field and velocity field for hopper half-angle $\theta_{w}=23^{\circ}, \phi=30^{\circ}$ and wall friction $\phi_{w}=11.5^{\circ}$. It is obvious that the stress discontinuity propagates over the whole region. Several kinds of distinct flow zones have also been predicted.

Figure 7 shows the effect of wall friction on wall pressure for $\theta_{w}=13^{\circ}$. It is noted that increasing the angle of wall friction will result in a decrease in $\sigma_{n n}$ while there seems to be no effect on the jump. Our knowledge of hopper pressure distribution indicates that the result is correct. In comparison with the finite element model by Schmidt and $\mathrm{Wu}$ [17], the method presented in this paper has a new feature that it is capable of simulating the stress discontinuity in the flow region.

\section{Conclusions}

A numerical technique has been developed to solve the hyperbolic partial differential equations arising from the formulation of the double-shearing theory for granular flows. The essential features of stress distribution and velocity pattern in hoppers are computed in the presence of stress discontinuity. The 


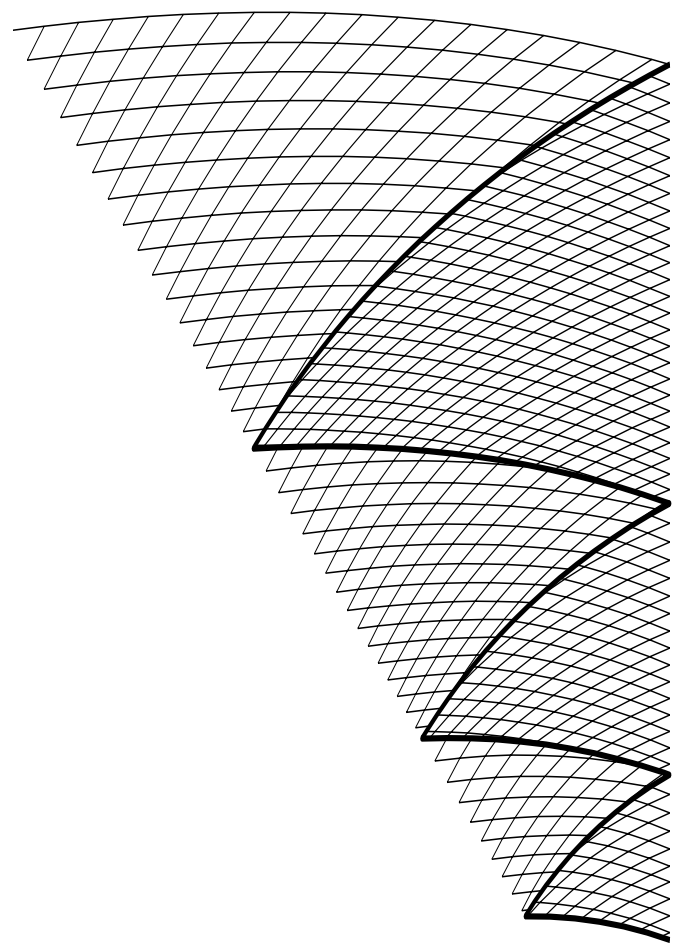

Figure 4: Characteristic mesh (thick lines indicate discontinuities). 


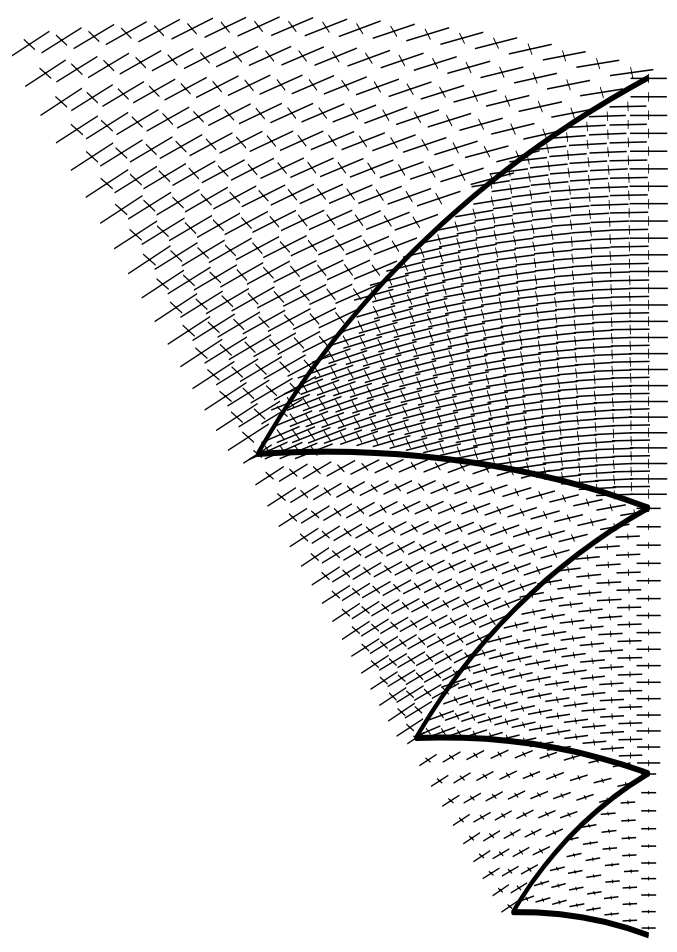

FiguRE 5: Principal stress field in the presence of discontinuity. 


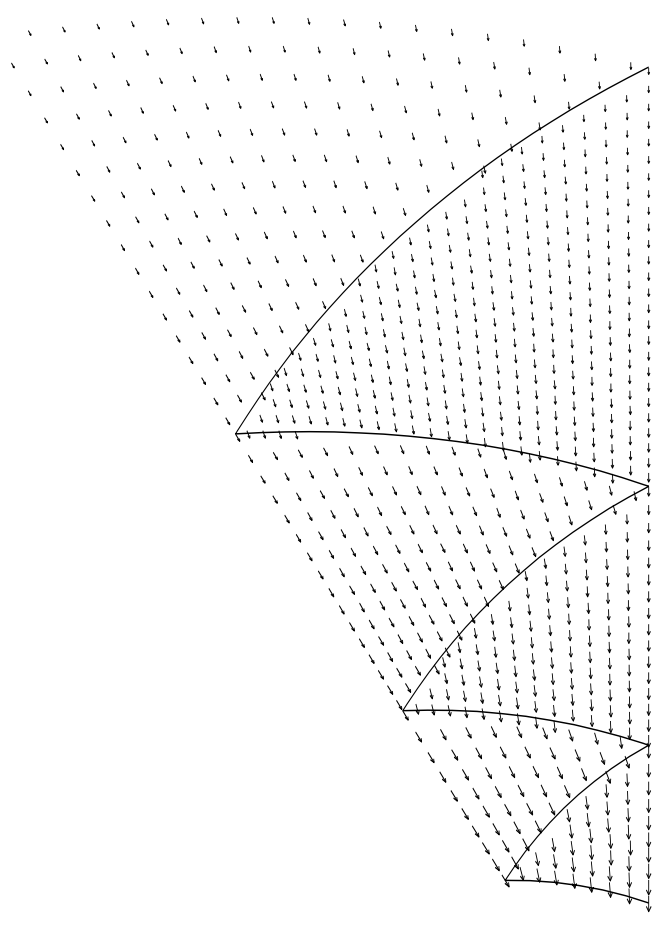

FiguRE 6: Velocity field in the presence of discontinuities. 


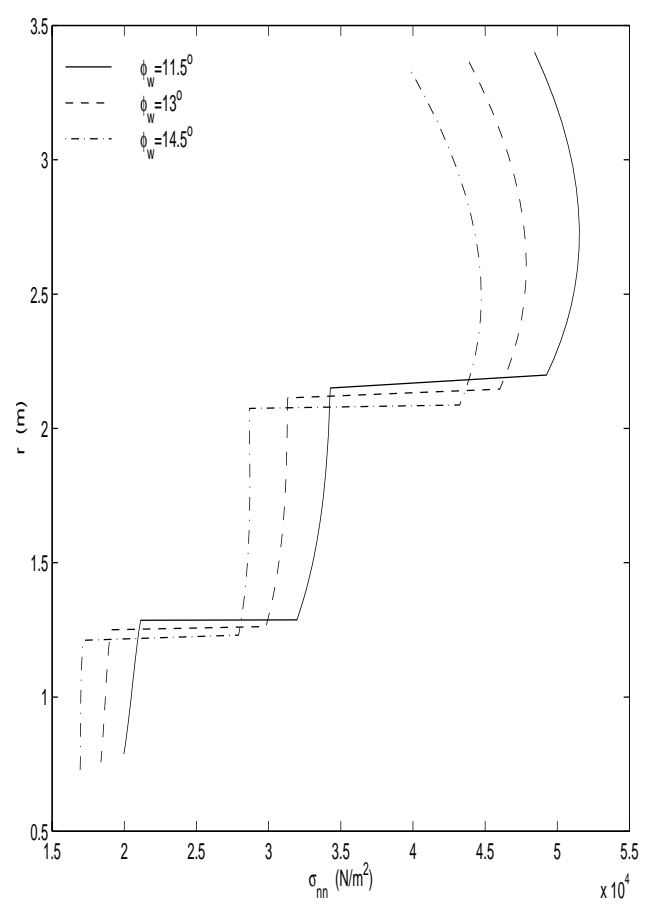

Figure 7: Wall pressure. 
development of the present numerical technique makes it possible to generate numerical solutions for many problems using the perfect plasticity theory based on the double shearing assumption. In addition, the numerical technique is capable of determining the stress discontinuity, which is important because it is one of the special features of granular flows which existing continuum models are not able to determine. However, it shall be mentioned that, the analysis given in the paper is for two-dimensional flows only. Although it is possible to generalise the method to axially symmetric problems, it is impossible or at least extremely difficult to generalise the method to general three-dimensional problems.

\section{References}

[1] B. R. Backman, C. B. Brown, P. W. Jowitt, and J. Munro. Statistical mechanics of granular materials. In M. Shahinpoor, editor Advances in the Mechanics and the Flow of Granular Materials, Vol.1, Trans Tech Publications, pages 259-272, 1984. Bulf Publishing Company. C1561

[2] A. Blinowski. On the dynamic flow of granular media. Archives of Mechanics, 30:27-34, 1978. C1562

[3] J. J. Brey, M. J. Ruiz-Montero, and F. Moreno. Steady uniform shear flow in a low density granular gas. Physical Review E, 55:2856+, 1997. C1562 
[4] C. S. Campbell. Rapid granular flows. Annu. Rev. Fluid Mech., 22:57-92, 1990. C1561

[5] P. Cleary and N. Stokes. Efficient collision detection for three dimensional super-ellipsoidal particles. In J. Noye, M. Teubner, and A. Gill, editors, Computational Techniques and Applications: CTAC97, 1998. World Scientific. C1561

[6] R. Collinson. Mathematical Models and Numerical Techniques for Plasticity Flows of Granular Media. PhD Thesis, Curtin University of Technology, 1998. C1561, C1570

[7] P. A. Cundall and O. D. L. Strack. A discrete numerical model for granular assemblies. Geotechnique, 29:47-65, 1979. C1561

[8] A. Goldshtein, M. Shapiro and C. Gutfinger. Mechanics of collisional motion of granular materials. Part 1. General hydrodynamic equations. J. Fluid Mechanics, 282:75-114, 1995. C1562

[9] J. M. Hill and Y. H. Wu. Plastic flows of granular materials of shear index n: Part I yield functions. J. Mech. Phys. Solids, 41:77-94, 1993. C1562

[10] M. A. Hopkins and H. H. Shen. A Monte Carlo simulation of a simple shear flow of granular materials. In M. Satake and J. T. Jenkins, editors, Micromechanics of Granular Materials, 1988. Elsevier Science Publications, Amsterdam. C1561 
[11] A. W. Jenike. Storage and flow of solids. Bulletin 123, University of Utah, Utah, 1964. C1561

[12] I. I. Kotchanova. Experimental and theoretical investigations on the discharge of granular materials from bins. Powder Technology, 4:32-37, 1970. C1561

[13] H. Matsuoka. Deformation and strength of granular materials based on the theory of compounded mobilized planes and spatial mobilized plane. In M. Shahinpoor, editor, Advances in the Mechanics and the Flow of Granular Materials (vol. 2), pages 814-836, 1984. Bulf Publishing Company. C1562

[14] A. W. Roberts. Bulk solids handling - recent developments and future directions. Int. J. of Bulk Solids Handling, 11:17-35, 1991. C1561

[15] M. C. Roco, V. Makhijani, and D. N. Ma. Probabilistic micromechanical model for two-dimensional slow particulate flow: Dry friction. Powder Technology, 60:223-243, 1990. C1561

[16] G. Rombach and J. Eibl. Granular flow of materials in silos. Int. J. of Bulk Solids Handling, 15:65-70, 1995. C1562

[17] L. C. Schmidt and Y. H. Wu. Prediction of dynamic wall pressures on silos. Int. J. of Bulk Solids Handling, 9:333-338, 1989. C1562, C1571

[18] A. J. M. Spencer. A theory of the kinematics of ideal soils under plane strain conditions. J. Mech. Phys. Solids, 12:337-351, 1964. C1562 
[19] O. R. Walton and R. L. Braun. Viscosity, granular temperature and stress calculations for shearing assemblies of inelastic, frictional disks. J. of Rheology, 30:949-980, 1985. C1561

[20] Y. H. Wu and M. Remias. Discrete element modelling of granular flows in silos. In Proceedings of IASTED Int. Conferenece on Modelling, Simulation and Optimization, Gold Coast, Qld, May 1996. C1561 